\title{
Development of an Interoperable GNSS Space Service Volume
}

\author{
Joel J. K. Parker, NASA Goddard Space Flight Center \\ Frank H. Bauer, FBauer Aerospace Consulting Services \\ Benjamin W. Ashman, NASA Goddard Space Flight Center \\ James J. Miller, NASA Headquarters \\ Werner Enderle, European Space Agency \\ Daniel Blonski, European Space Agency
}

\section{BIOGRAPHIES}

Joel J. K. Parker is the Positioning, Navigation, and Timing (PNT) Policy lead at NASA Goddard Space Flight Center, where he develops and promotes civil space use of GNSS on behalf of NASA Space Communications and Navigation (SCaN). He has worked as an Aerospace Engineer at NASA since 2010.

Frank H. Bauer supports NASA with expertise in spacecraft systems engineering, Guidance Navigation and Control (GN\&C), space-borne GNSS, and spacecraft formation flying. He previously served as NASA's Chief Engineer for Human Spaceflight Exploration beyond low Earth Orbit and as Chief of the GN\&C Division at NASA's Goddard Space Flight Center.

Benjamin W. Ashman is an Aerospace Engineer at the NASA Goddard Space Flight Center, where he supports numerous space communication and navigation efforts, most recently as part of the OSIRIS-REx navigation team. His research has primarily been focused on space applications of GPS since starting at the agency in 2015.

James J. Miller is Deputy Director of Policy \& Strategic Communications within the Space Communications and Navigation Program at the NASA Headquarters. His duties include advising NASA leadership on Positioning, Navigation, and Timing (PNT) policy and technology, and is also the Executive Director of the National Space-based PNT Advisory Board.

Werner Enderle is the Head of the Navigation Support Office at ESA's European Space Operations Center (ESOC) in Darmstadt, Germany. Previously, he worked at the European GNSS Authority (GSA) as the Head of System Evolutions for Galileo and EGNOS and he also worked for the European Commission in the Galileo Unit. Since more than 20 years, he is involved in activities related to the use of GPS/GNSS for space applications. He holds a doctoral degree in aerospace engineering from the Technical University of Berlin, Germany.

Daniel Blonski is Navigation System Performance Engineer at the European Space Research and Technology Centre of the European Space Agency in Noordwijk, NL, where he is contributing to the development of the European Navigation Systems as a member of the ESA Directorate of Navigation.

\begin{abstract}
Global Navigation Satellite Systems (GNSS), now routinely used for navigation by spacecraft in low Earth orbit, are being used increasingly by high-altitude users in geostationary orbit and high eccentric orbits as well, near to and above the GNSS constellations themselves. Available signals in these regimes are very limited for any single GNSS constellation due to the weak signal strength, the blockage of signals by the Earth, and the limited number of satellites. But with the recent development of multiple GNSS constellations and ongoing upgrades to existing constellations, multi-GNSS signal availability is set to improve significantly. This will only be achieved if these signals are designed to be interoperable and are clearly documented and supported.
\end{abstract}

All satellite navigation constellation providers are working together through the United Nations International Committee on GNSS (ICG) to establish an interoperable multi-GNSS Space Service Volume (SSV) for the benefit of all GNSS space users. The multi-GNSS SSV represents a common set of baseline definitions and assumptions for high-altitude service in space, documents the service provided by each constellation, and provides a framework for continued support for space users. This 
paper provides an overview of the GNSS SSV concept, development, status, and achievements within the ICG. It describes the final adopted definition and performance characteristics of the GNSS SSV, as well as the numerous benefits and use cases enabled by this development. Extensive technical analysis was also performed to illustrate these benefits in terms of signal availability, both on a global scale, and for multiple distinct mission types. This analysis is summarized here and presented in detail in a companion paper by Enderle, et al.

\section{INTRODUCTION}

Global navigation satellite systems (GNSS), which were originally designed to provide positioning, velocity, and timing services for terrestrial users, are now increasingly utilized for autonomous navigation in space as well. Historically, most space users have been located at low altitudes, where GNSS signal reception is similar to that on the ground. More recently, however, users are relying on these signals at high altitudes, near to or above the GNSS constellations themselves. High-altitude applications of GNSS are more challenging than terrestrial or low Earth orbit (LEO) applications due to a number of factors. As shown in Figure 1, a significant portion of the GNSS Earth-pointing main-lobe signal is occulted by the Earth. Some of this signal, however, passes the limb of the Earth and is received by high-altitude user spacecraft beyond, but with reduced coverage. Because of the longer path length, signal strength is up to an order of magnitude weaker, as compared to that received by LEO or terrestrial users. Finally, from the point of view of the receiving spacecraft, the GNSS satellites are clustered in a smaller portion of the sky, reducing the geometric diversity of the received signals and, thus, reducing precision of the final navigation solution.

The first flight experiments tracking the US Global Positioning System (GPS) above the constellation were launched in 1997 and successfully demonstrated that such signals could be tracked and acquired [1-2]. Later experiments, such as AMSAT OSCAR-40 [3] in 2001 and the later GIOVE-A GPS experiment [4] in 2013 collected on-orbit tracking data over an extended period and revealed performance characteristics of the GPS signal structure itself for high-altitude users. Operational highaltitude use has been documented as early as 2000 for users of GPS at geostationary altitude [5], and for multi-GNSS using both GPS and Russia's GLONASS starting in 2007 [6]. Recent published examples include NASA's Magnetospheric Multiscale (MMS) mission [7], which successfully uses GPS at 40\% of lunar distance, and the US Geostationary Operational Environmental Satellite-R (GOES-R) series of weather satellites [8]. A major finding of the AMSAT OSCAR-40 experiment was that in addition to the inherent challenges of high-altitude GNSS use, the characteristics of the transmitted signals reaching this regime were also changing as the design of the GPS satellites evolved. This prevented accurate mission planning, as designers could not make assumptions about future GPS signal availability and power. The GPS Space Service Volume (SSV) [9] was created to address this issue. The GPS SSV specifies the volume of GPS service around the Earth and establishes formal requirements for signal received power, availability, and accuracy. Thus, the SSV defines a guaranteed lower limit on the GPS signal capabilities in this region, which can be employed by space mission planners to design on-board receiver equipment and simulate mission performance.

The recent and ongoing expansion of GNSS beyond just one or two constellations opens up new opportunities for high-altitude users. There will soon be four operational global constellations and two regional augmentations, respectively: the US' Global Positioning System (GPS), Russia's GLONASS, Europe's Galileo, China’s BeiDou (BDS), Japan's Quasi-Zenith Satellite System (QZSS), and India's Navigation with Indian Constellation (NavIC). Table 1 provides an overview of these systems and their high-level characteristics. When combined, this "super-constellation" of 100+ GNSS satellites has the potential to greatly improve the signal coverage available in the high-altitude regime, as well as to increase the diversity of system architectures, signal frequencies, and signal geometries. Together, these constellations have the potential to improve overall performance and resiliency for users. But this potential can only be realized if these systems and signals are designed to be interoperable, and if they are designed with sufficient signal performance to benefit high-altitude users. All satellite navigation constellation providers are now working together through the United Nations (UN) International Committee on GNSS (ICG) to establish a coordinated, interoperable multi-GNSS SSV with the goal to ensure that these benefits are extended to the emerging class of high-altitude space users that wish to exploit the use of multi-GNSS navigation and timing.

Table 1. Overview of global and regional navigation satellite systems 


\begin{tabular}{|c|c|c|c|c|c|c|c|c|}
\hline 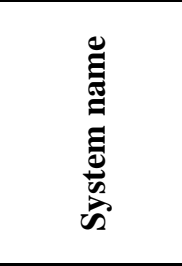 & : & 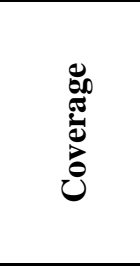 & $\sum_{\tilde{D}}^{n}$ & 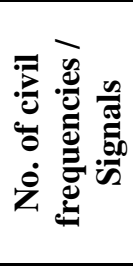 & 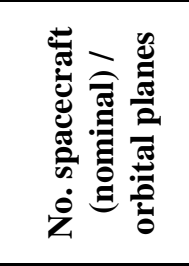 & 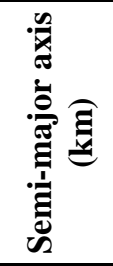 & 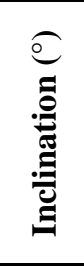 & 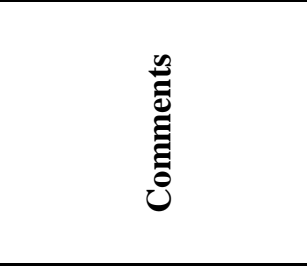 \\
\hline GPS & USA & Global & Operational & $3 / 4$ & $27 / 6$ & 26560 & 55 & \\
\hline GLONASS & Russia & Global & Operational & $2 / 6$ & $24 / 3$ & 25510 & 64.8 & \\
\hline Galileo & $\begin{array}{l}\text { European } \\
\text { Union }\end{array}$ & Global & Operational & $5 / 10$ & $24 / 3$ & 29600 & 56 & $\begin{array}{l}\text { Initial Service: } 2016 \\
\text { FOC }^{5} \text { planned: } 2020\end{array}$ \\
\hline BDS & China & Global & $\begin{array}{c}\text { Operational } \\
\text { (Regional) } \\
\text { In build-up } \\
\text { (Global) }\end{array}$ & $3 / 5$ & $\begin{array}{c}\text { MEO }^{1}: 24 / 3 \\
\text { IGSO }^{2}: 3 / 3 \\
\text { GEO }^{3}: 5 / 1\end{array}$ & $\begin{array}{l}27906 \\
42164 \\
42164\end{array}$ & $\begin{array}{c}55 \\
55 \\
0\end{array}$ & $\begin{array}{l}\text { Service planned: } \\
\text { Regional FOC: } 2012 \\
\text { Global Initial } \\
\text { Service: } 2018 \\
\text { FOC: } 2020 \\
\end{array}$ \\
\hline QZSS & Japan & $\begin{array}{c}\text { Regional } \\
\text { (Japan) }\end{array}$ & In build-up & $4 / 7$ & $\begin{array}{l}\mathrm{HEO}^{4}: 3 / 3 \\
\text { GEO: } 1 / 1\end{array}$ & 42164 & $\begin{array}{c}40 \\
0\end{array}$ & $\begin{array}{l}\text { Service planned: } \\
2018\end{array}$ \\
\hline NavIC & India & $\begin{array}{c}\text { Regional } \\
\text { (India) }\end{array}$ & In build-up & $2 / 2$ & $\begin{array}{l}\text { IGSO: } 4 / 2 \\
\text { GEO: } 3 / 1\end{array}$ & 42164 & $\begin{array}{c}29 \\
0\end{array}$ & $\begin{array}{l}\text { Service planned: } \\
2018\end{array}$ \\
\hline \multicolumn{9}{|c|}{$\begin{array}{l}{ }^{1} \text { medium Earth orbit } \\
{ }^{2} \text { inclined geosynchronous orbit } \\
{ }^{3} \text { geostationary orbit } \\
{ }^{4} \text { high eccentric orbit } \\
\text { 5full operational capability }\end{array}$} \\
\hline
\end{tabular}

\section{ICG AND THE SSV DEVELOPMENT PROCESS}

In the early 2000's, several bilateral meetings were held to discuss GNSS signal interoperability. These included meetings with the US and Russia on GPS/GLONASS interoperability and with the US and Europe on GPS/Galileo. These bilateral GNSS discussions ultimately became a multilateral dialog in 2005 when the United Nations formed the International Committee on GNSS (ICG). The ICG was created "to encourage and facilitate compatibility, interoperability ${ }^{1}$ and transparency between all the satellite navigation systems, to promote and protect the use of their open service applications and thereby benefit the global community." [11] To maximize the utility of GNSS for high-altitude space users, coordination among all GNSS service providers is taking place within two working groups: Working Group B (WG-B) on "Enhancement of GNSS Performance, New Services and Capabilities", which leads SSV development, coordination, and outreach; and Working Group S on "Systems, Signals and Services", which coordinates the underlying GNSS signal interoperability [12].

The WG-B team undertook a series of activities in support of Task 3 of the WG-B Work Plan [13], which directs it to continue "the implementation of an interoperable GNSS Space Service Volume and provide recommendations to Service Providers regarding possible evolution needs arising from users/application developers." To further emphasize SSV critical strategies, WG-B also forwarded a series of recommendations, shown later in this section, to the ICG delegates for formal adoption. All initiatives were coordinated within the semi-annual ICG and intersessional meetings and in monthly WG-B teleconferences, and were conducted by the WG-B international team.

The following represents a snapshot of activities undertaken in support of the WG-B Work Plan:

- SSV Definition/Assumption Maturation: Development of standard definitions, ground rules, and assumptions of the multi-GNSS SSV and related concepts to support coordinated technical analyses across the international team and to be employed for formal SSV specification efforts.

- Constellation-Specific SSV Performance Data: Documenting and publishing the SSV performance metrics for each individual constellation in standard template form. This data is summarized in Table 2 and Table 3.

\footnotetext{
${ }^{1}$ The ICG defines interoperability as "the ability of global and regional navigation satellite systems, and augmentations and the services they provide, to be used together to provide better capabilities at the user level than would be achieved by relying solely on the open signals of one system.” [10]
} 
- Multilateral SSV Analysis: An international analysis effort to characterize single-constellation and multipleconstellation performance expectations within the SSV, using both a coverage grid (global) approach, and a suite of example mission profiles. The results of these analyses are captured in the GNSS SSV Booklet and in a set of coordinated conference and journal papers and will serve as a reference for space mission analysts.

- GNSS SSV Booklet: Development of an authoritative public ICG document that documents the multi-GNSS SSV, including coordinated definitions; benefits and use cases of the interoperable GNSS SSV; constellation-specific SSV signal performance data; and the results of technical analysis to estimate the performance capabilities of an interoperable multi-GNSS SSV. This booklet will be updated with new GNSS provider data and additional information as needed.

- SSV Capabilities Outreach: Coordination of a joint international outreach activity, targeted at space agencies, researchers, spacecraft mission designers, navigation engineers, GNSS providers, and others, to ensure that the capabilities and benefits of the interoperable GNSS SSV are understood, supported, and utilized.

The products from these WG-B activities are included in the SSV Booklet [14], which captures the definitions, template data and analysis results, and will be summarized in the outreach presentations and conference and journal papers. A major SSV milestone was reached at the 2015 ICG-10 meeting held in Boulder, Colorado, US, where all 6 GNSS providers (China, Europe, India, Japan, Russia, and the United States) formally submitted their SSV template data, and a recommendation was formally adopted to develop the Booklet. The multi-GNSS SSV Booklet is now complete and is awaiting final publication by the UN, expected by Fall 2018. The Booklet will be available to the public and will be available on several international GNSS web sites. The internationally-coordinated SSV analysis effort, which presents a conservative technical baseline for expected space user performance, is complete, has been adopted by all providers, and is documented in the Booklet and in a companion paper by Enderle, et al [15]. Outreach activities have commenced by members of the WG-B team, who presented the SSV initiative in a paper by Bauer, et al. in January 2017 [16], conducted a panel discussion at the Munich Satellite Navigation Summit in March 2017, and are planning further outreach events upon publication of the Booklet.

Four formal WG-B recommendations were adopted and endorsed by the ICG to encourage continued development, support, and expansion of the multi-GNSS SSV concept. For the community of GNSS providers, the following recommendations are aimed at continuing development of the SSV and providing the user community adequate data to utilize it:

- ICG/WGB/2014 Rec. 2: GNSS providers are recommended to support the SSV outreach by making the booklet on "Interoperable GNSS Space Service Volume" available to the public through their relevant websites once the booklet is available. [17]

- ICG/WGB/2016 Rec. 1: Service Providers, supported by Space Agencies and Research Institutions, are encouraged to define the necessary steps and to implement them in order to support SSV in future generations of satellites. Service Providers and Space Agencies are invited to report back to WG-B on their progress on a regular basis. [18]

- ICG/WGB/2016 Rec. 3: GNSS providers are invited to consider for the future, to provide the following additional data if available:

- GNSS transmit antenna gain patterns for each frequency, measured by antenna panel elevation angle at multiple azimuth cuts, at least to the extent provided in each constellation's SSV template.

- In the long term, GNSS transmit antenna phase centre and group delay patterns for each frequency. [18]

For the user community, there is one recommendation, to ensure that the full capabilities of the multi-GNSS SSV can be utilized:

- ICG/WGB/2013 Rec. 1: The authors encourage the development of interoperable multi-frequency space borne GNSS receivers that exploit the use of GNSS signals in space. [19]

\section{DEFINITION OF THE MULTI-GNSS SPACE SERVICE VOLUME}

The following is the formal definition of the multi-GNSS SSV, as adopted by the ICG WG-B.

The GNSS Space Service Volume (SSV) is defined in the context of the SSV Booklet [14] as the region of space extending from 3,000 km to 36,000 km altitude, where terrestrial GNSS performance standards may not be applicable. GNSS system service in the SSV is defined by three key parameters:

- Pseudorange accuracy

- Minimum received power

- Signal availability 
The SSV covers a large range of altitudes, and the GNSS performance will degrade with increasing altitude. In order to allow for a more accurate reflection of the performance variations, the SSV itself is divided into two distinct areas that have different characteristics in terms of the geometry and quantity of signals available to users in those regions:

1. Lower Space Service Volume for Medium Earth Orbits: 3,000-8,000 km altitude. This area is characterized by reduced signal availability from a zenith-facing antenna alone, but increased availability if both a zenith and nadirfacing antenna are used.

2. Upper Space Service Volume for Geostationary and High Earth Orbits: $8,000-36,000 \mathrm{~km}$ altitude. This area is characterized by significantly reduced signal received power and availability, due to most signals traveling across the limb of the Earth.

Users with adequate antenna and signal processing capabilities will also be able to process GNSS signals above the identified altitude of $36,000 \mathrm{~km}$.

The relevant regions of the GNSS SSV are depicted in Figure 1, along with the altitude ranges of the contributing GNSS constellations that are located in Medium-Earth Orbit (MEO). It is noted that some GNSS also offer satellites at Geostationary Orbits (GEO) and/or Inclined Geosynchronous Orbits (IGSO).

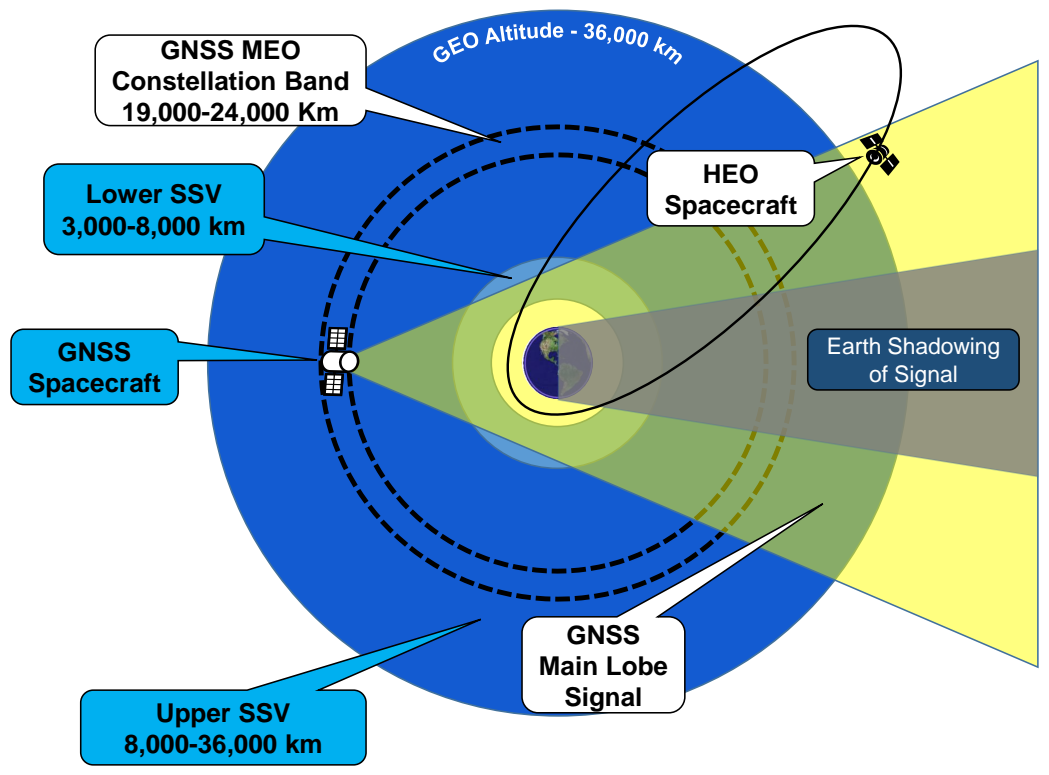

Figure 1. The GNSS Space Service Volume and its regions

The characterization of the SSV performance of an individual GNSS constellation relates at a minimum to the characterization of the following three parameters for every ranging signal:

1. Pseudorange Accuracy: Since users in the SSV do not typically generate Position, Velocity and Time (PVT) solutions using multiple simultaneous GNSS measurements, this instead measures the error in the ranging signal itself. This relates to the orbit determination and clock stability errors, and additional systematic errors.

2. Received Signal Power: This is the minimum user-received signal power obtained by a space user in the relevant orbit, assuming a $0 \mathrm{dBic}$ user antenna. Generally, this power is calculated at the highest altitude in the given SSV region.

3. Signal Availability: Signal availability is calculated as the percent of time that GNSS signals are available for use by a space user. It is calculated both as the availability of a single signal in view, and as the availability of four signals in view, to capture the various requirements of space users. In both cases, in order to declare a signal available, it needs to be both: 
a. received at a signal power level higher than the minimum specified for SSV users, and

b. observed with a user range error smaller than the maximum user range error specified for SSV users.

The signal availability is measured as a metric over a shell at a given altitude (e.g. at 36,000 km) and is generated as a statistic over both location and time. The exact calculation used for this metric by an individual GNSS constellation is specified explicitly in Annex A of the SSV Booklet.

A sub-metric to Signal Availability is Maximum Outage Duration, defined as the maximum duration when a space user at a particular orbit will not obtain availability for at least one single signal or at least four signals simultaneously, depending on the exact metric being calculated. The definition of maximum outage duration is closely linked to the definition of signal availability.

These three parameters characterize at a minimum the contribution of an individual GNSS to an interoperable GNSS SSV. In addition to these parameters, constellation service providers may identify additional parameters useful to characterize their particular contribution to the interoperable GNSS SSV.

\section{INDIVIDUAL CONSTELLATION CONTRIBUTIONS TO GNSS SSV}

A primary outcome of the multi-GNSS SSV development process was the publication of expected performance in the SSV by each GNSS constellation provider, and thus its contribution to the total multi-GNSS SSV. To convey a consistent set of capabilities across all constellations, an SSV capabilities template was completed by each GNSS service provider to capture their contributions to each of the parameters identified in the previous section. The full text of these completed templates, along with appropriate context, is available in Annex A of the SSV Booklet. Table 2 and Table 3 contain a summary of the key data, focusing specifically on the Upper SSV and the L1/E1/B1 ("L1") and L5/L3/E5/B2 ("L5") bands. The full templates contain data for the Lower SSV and the L2/E6 band, along with other details.

Table 2. Individual Constellation SSV Template Data-Received Power and Upper SSV Availability

\begin{tabular}{|c|c|c|c|c|c|}
\hline \multirow[t]{2}{*}{ Band } & \multirow[t]{2}{*}{ Constellation } & \multicolumn{2}{|c|}{$\begin{array}{c}\text { Minimum Received Civilian } \\
\text { Signal Power }\end{array}$} & \multicolumn{2}{|c|}{$\begin{array}{c}\text { Upper SSV } \\
\text { Signal Availability }(\%)\end{array}$} \\
\hline & & $\begin{array}{l}\text { OdBi RCP } \\
\text { antenna at } \\
\text { GEO }(\mathrm{dBW})\end{array}$ & $\begin{array}{l}\text { Reference } \\
\text { off-boresight } \\
\text { angle }\left(^{\circ}\right)\end{array}$ & $\begin{array}{l}\text { At least } 1 \\
\text { signal }\end{array}$ & $\begin{array}{l}4 \text { or more } \\
\text { signals }\end{array}$ \\
\hline \multirow[t]{5}{*}{ L1/E1/B1 } & GPS & $\begin{array}{l}-184(\mathrm{C} / \mathrm{A})^{1} \\
-182.5(\mathrm{C})^{2}\end{array}$ & 23.5 & 80 & $\overline{1}$ \\
\hline & GLONASS & -179 & 26 & 93.9 & 7.0 \\
\hline & Galileo & -182.5 & 20.5 & 64 & 0 \\
\hline & BDS & $\begin{array}{l}-184.2(\mathrm{MEO})^{3} \\
-185.9(\mathrm{I} / \mathrm{G})^{4}\end{array}$ & $\begin{array}{l}25 \\
19\end{array}$ & 97.40 & 24.10 \\
\hline & QZSS & -185.5 & 22 & 54 & N/A \\
\hline \multirow[t]{6}{*}{ L5/L3/E5/B2 } & GPS & -182 & 26 & 92 & 6.5 \\
\hline & GLONASS & -178 & 34 & 99.9 & 60.3 \\
\hline & Galileo & $\begin{array}{l}-182.5 \text { (E5b) } \\
-182.5 \text { (E5a) }\end{array}$ & $\begin{array}{l}22.5 \\
23.5\end{array}$ & $\begin{array}{l}80 \\
86\end{array}$ & $\begin{array}{l}0 \\
0\end{array}$ \\
\hline & BDS & $\begin{array}{l}-182.8(\mathrm{MEO}) \\
-184.4(\mathrm{I} / \mathrm{G})\end{array}$ & $\begin{array}{l}28 \\
22\end{array}$ & 99.90 & 45.40 \\
\hline & QZSS & -180.7 & 24 & 54 & N/A \\
\hline & NavIC & -184.54 & 16 & 36.90 & 0.60 \\
\hline \multicolumn{6}{|l|}{$\begin{array}{l}{ }^{1} \mathrm{~L} 1 \mathrm{C} / \mathrm{A} \text { signal } \\
{ }^{2} \mathrm{~L} 1 \mathrm{C} \text { signal }\end{array}$} \\
\hline
\end{tabular}

Table 3. Individual Constellation SSV Template Data-Pseudorange Accuracy

\begin{tabular}{|l|llllll|}
\hline Constellation & GPS & GLONASS & Galileo & BDS & QZSS & NavIC \\
\hline Pseudorange accuracy & $0.8 \mathrm{~m}$ & $1.4 \mathrm{~m}$ & $1.1 \mathrm{~m}$ & $2.5 \mathrm{~m}$ & $2.6 \mathrm{~m}$ & $2.11 \mathrm{~m}$ \\
\hline
\end{tabular}


Note that the SSV template data outlined here and in the Booklet represent the service documented by each individual GNSS service provider, either by formal specification or by characterization and analysis. This data is intended to be conservative, to be used as a baseline estimate of the capability provided to the multi-GNSS SSV by each constellation for mission planning purposes, and based on constellation-specific assumptions as documented in Annex A of the SSV Booklet [14]. On-orbit flight results will vary based on mission-specific geometry, receiver sensitivity, time-dependent service characteristics, and other factors. In particular, only service provided by the main-lobe signal services are captured here; the boundary of this main-lobe service is documented in the templates as the reference off-boresight angle.

\section{ESTIMATED PERFORMANCE OF THE MULTI-GNSS SSV}

To evaluate the technical benefit of the combined multi-GNSS SSV, the ICG WG-B performed a collaborative multilateral simulation of the GNSS single-constellation and multiple-constellation performance expectations in the SSV, based on the individual constellation signal characteristics. Both global and mission-specific analyses were performed, to capture both overall performance characteristics and representative performance in different orbit regimes. Navigation performance in the SSV is primarily characterized by three properties: pseudorange accuracy, minimum received signal power, and signal availability. The focus of these simulations was on signal availability, which serves as a proxy for navigation capability, and on the L1 and L5 bands.

As an example of the potential improvement in signal availability in the multi-GNSS SSV, Table 4 shows summarized results of the global performance analysis in the upper SSV. Global SSV performance was estimated by simulating signal availability at a fixed grid of points in space, at 36,000 km altitude, taking into account the path loss between transmitter and receiver spacecraft. The GNSS constellations themselves were simulated in accordance with the configurations and performance characteristics shown in Table 1 and Table 2. An available signal was considered to be one for which the receiver has an unobstructed line of sight and is able to detect the signal with sufficient strength, represented as a carrier to noise spectral density $\left(\mathrm{C} / \mathrm{N}_{0}\right)$ value. The availability was calculated at all points in each grid over the simulation duration, and the percent availability was calculated over the interval and by averaging over all grid locations. Maximum continuous outage duration was also recorded for each grid point and is quoted in the results at the worst-case location. These results are intended to be conservative estimates based on the main-lobe signals described in Table 2, and do not consider other factors that may be specific to any particular mission user. For more details on the methods, assumptions, and results of this analysis, see the companion paper by Enderle, et al. [15] and the SSV Booklet [14].

Table 4. Summarized Upper SSV Global Performance Estimates (20 dB-Hz C/NO threshold)

\begin{tabular}{|c|c|c|c|c|c|}
\hline \multirow[t]{2}{*}{ Band } & \multirow[t]{2}{*}{ Constellation } & \multicolumn{2}{|c|}{ At least 1 signal } & \multicolumn{2}{|c|}{4 or more signals } \\
\hline & & Avail. (\%) ${ }^{1}$ & $\operatorname{MOD}(\min )^{2}$ & Avail. $(\%)^{1}$ & MOD $(\min )^{2}$ \\
\hline \multirow[t]{3}{*}{ L1/E1/B1 } & Global systems & $78.5-94$ & $48-111$ & $0.6-7$ & * \\
\hline & QZSS & 0 & $* 3$ & 0 & * \\
\hline & Combined & 99.9 & 33 & 89.8 & 117 \\
\hline \multirow{3}{*}{ L5/L3/E5a/B2 } & Global systems & $93.4-99.9$ & 7_* & $4.2-60.3$ & $218-*$ \\
\hline & Regional systems & $1-30.5$ & $*$ & $0-1.5$ & * \\
\hline & Combined & 100 & $\mathbf{0}$ & 99.9 & 15 \\
\hline
\end{tabular}

As shown in Table 4, at a receiver threshold of $20 \mathrm{~dB}-\mathrm{Hz}$, four-signal $\mathrm{L} 1$ availability in the Upper SSV with any individual constellation is very low, reaching only $7 \%$. When the systems are used together, however, four-signal availability reaches nearly $90 \%$, allowing kinematic solutions throughout the SSV with less than two hours of outage, and indicating the importance of using specialized high-altitude receivers and high-gain antennas. Furthermore, one-signal visibility is at nearly $100 \%$ and is greater than $90 \%$ at $25 \mathrm{~dB}-\mathrm{Hz}$, indicating nearly continuous signal coverage for the purposes of provided measurements to a navigation filter, or providing time updates. The abundance of signals available in an interoperable multi-GNSS SSV greatly reduces constraints for navigation at high altitudes.

\section{USER BENEFITS AND APPLICATIONS}

The development of a multi-GNSS SSV will have numerous benefits for high-altitude space users, including improved navigation performance, enhanced operations, and mission-enabling technology advancement. 
The most direct benefit to users is the improvement of spacecraft navigation performance in high-altitude regimes through a greater number of available signals, improved relative geometry, and reduced signal outages. In the global analysis performed as part of this development, the availability of four simultaneous L1 signals to support the generation of a kinematic position solution at GEO was improved from a maximum of $7 \%$ for any single constellation to $90 \%$ for all constellations combined, using moderate receiver performance assumptions [14]. While not specifically addressed in the current analysis, combining multiple constellations, especially those of different orbit regimes (e.g., MEO and GEO), will also reduce dilution of precision (DOP), improving overall navigation accuracy. Finally, the multi-GNSS SSV will reduce and even eliminate the maximum outage duration between GNSS measurements. This would, in turn, reduce the need for precise, on-board clocks to maintain accurate timing during the outage period.

The multi-GNSS SSV promises to be transformative in the operation of high altitude spacecraft. The improved navigation performance and signal availability provided will enable increased spacecraft autonomy, thereby reducing the overall required ground interactions for navigation and, in turn, reducing ground operational costs. The use of multiple, independent GNSS signals, frequencies, and constellations will increase operational robustness. And with fewer ground-based ranging requests and mission support needs for navigation, mission architectures can be simplified and even standardized. International efforts to establish a multi-GNSS SSV have raised the visibility of the high-altitude space user community, encouraging the development and availability of new multi-GNSS space receivers that can take advantage of high altitude capabilities. As a result, new mission concepts that rely on continuous, precise on-board timing and navigation will become feasible. Specific examples include advanced Earth weather observation, precise and autonomous formation flying, robust cislunar exploration in the absence of ground contact, satellite servicing, and improved colocation of GEO satellites, as described below.

Earth weather observation. The United States' Geostationary Operational Environmental Satellite R-series spacecraft (GOESR) collects weather observations continually, with outages of less than 2 hours per year, even with daily low-thrust stationkeeping maneuvers [20]. To accomplish this, they rely on nearly-continuous GPS signals in the SSV. Likewise, Russia's Elektro-L weather satellites have been using combined GPS/GLONASS signals in GEO since 2011. Increased capability in the multi-GNSS SSV will support the next-generation of Earth weather observation satellites with even more stringent navigation requirements.

Precision formation flying. The upcoming European Proba-3 solar occultation mission seeks to observe the Sun's corona by flying a solar-occulting spacecraft and an observing spacecraft in precise formation, in a highly-elliptical Earth orbit [16]. The highly-precise relative positioning of the two spacecraft will rely on highly-available GNSS signals up to approximately 60,000 $\mathrm{km}$ altitude.

Cislunar trajectories. Launch vehicle upper stages and cislunar exploration missions travel well beyond GEO altitude, with some traveling all the way to lunar distance. GNSS is planned to be used by these vehicles for its high accuracy and high cadence, which improves insertion accuracy when returning to Earth. Weak-signal receivers enable use of GNSS signals at extremely long distances as well, potentially allowing for use as a supplemental measurement source in lunar orbit to increase vehicle navigation responsiveness and autonomy.

Satellite servicing. Satellite servicing missions are being developed for spacecraft at GEO, where they will need to autonomously rendezvous with their target spacecraft. The precision and autonomy required for this type of mission will require continuous precise GNSS signals to be available.

New Concepts for GEO Co-location. The most highly sought-after orbits for commercial users are in the GEO belt, where the current number of spacecraft is limited by the longitude spacing requirements put in place to avoid collisions. With GNSS, these spacecraft could reduce relative navigation errors, recover quickly from maneuvers, and reduce burden on the ground operations infrastructure, potentially resulting in more efficient use of available space at GEO.

\section{CONCLUSIONS}

The number and scope of GNSS based space applications has grown significantly since the first GNSS space receiver was flown in the early 1980's. The vast majority of space users are operating in Low-Earth Orbit where use of GNSS receivers has become routine. However, the use of GNSS has expanded to other orbit regimes like Geostationary Orbits (GEO) and High Eccentric Orbits (HEO) but have been very limited due to the challenges involved, including much weaker signals, reduced geometric diversity, and limited signal availability. The GNSS Space Service Volume (SSV) was defined to provide a framework for documenting and specifying GNSS constellation performance for these users, up to an altitude of 36,000 km. 
The United Nations International Committee on GNSS (ICG) Working Group B (WG-B) has worked on a collaborative basis to publicize the performance of each GNSS constellation in the SSV, and to promote the establishment of an interoperable multi-GNSS SSV in which all existing GNSS constellations can be utilized together to improve mission performance.

This effort has resulted in an internationally-agreed definition of the multi-GNSS SSV and captures the SSV performance contributions of each individual GNSS constellation in terms of pseudorange accuracy, minimum received signal power, and signal availability. In addition, simulations show the performance benefits of the combined systems. In particular, there are significant availability improvements over any individual constellation when all GNSS constellations are employed. Within the Upper SSV, single-signal availability reaches $99 \%$ for the L1 band with a $20 \mathrm{~dB}-\mathrm{Hz} \mathrm{C} / \mathrm{N} 0$ threshold and zero-gain antenna, and four-signal availability jumps from a maximum of $7 \%$ for any individual constellation to $90 \%$ with all. Further, similar benefits are shown explicitly for geostationary, highly-elliptical, and lunar use cases.

There are many benefits to an interoperable multi-GNSS SSV, including increased signal availability for high-altitude users over that provided by any individual constellation alone, increased geometric diversity and thus accuracy in the final navigation solution, increased responsiveness and potential autonomy due to reduced signal outages, and increased resiliency due to the diversity of signals and constellations used. These benefits are truly enabling for classes of emerging advanced users, including ultra-stable remote sensing from geostationary orbit (GEO), agile and responsive formation flying, and more efficient utilization of valuable slots in the GEO belt.

The ICG WG-B plans to continue to develop the multi-GNSS SSV concept and analyses, including evolving the analysis to include more consideration of geometric diversity, accurate GNSS transmit antenna patterns and characteristics, improved receiver mission and equipment data, identification of further benefits and use cases, and studying advanced "beyond-SSV" applications. The development of the multi-GNSS SSV was truly an international cooperative effort by all GNSS providers. The resulting high-altitude GNSS capability will enhance spacecraft navigation, enable new mission concepts, and advance spaceborne science, for the benefit of humanity.

\title{
ACKNOWLEDGEMENTS
}

Sincere thanks go to all that supported the development of the interoperable GNSS Space Service Volume. Special recognition goes to the GNSS/RNSS providers including the: US Air Force GPS Directorate, the Russia GLONASS team, the European Commission and EU Galileo team, China's BDS team, Japan's QZSS team, and India's NavIC team. Instrumental in the multiGNSS SSV development were the leaders and international delegates from the UN International Committee on GNSS, including the ICG Secretariat Sharafat Gadimova and the relevant ICG working groups. In particular, the Working Group B "Enhancement of GNSS Performance, New Services and Capabilities" leaders and SSV subject matter experts, acknowledged in Annex F of the UN ICG booklet, transformed the GNSS SSV from vison to reality. The contributions of these experts will enable untold space missions that will extend humanity's reach for the stars, its understanding of the planet Earth, and its critical protection of people and property.

\author{
ACRONYMS \\ BDS BeiDou System (China) \\ $\mathrm{C} / \mathrm{N}_{0} \quad$ Carrier Power to Noise Spectral Density \\ FOC Full Operational Capability \\ GEO Geostationary Earth Orbit \\ GLONASS Global Navigation Satellite System (Russia) \\ GNSS Global Navigation Satellite Systems \\ GOES Geostationary Operational Environmental Satellite \\ GPS Global Positioning System (US) \\ ICG UN International Committee on GNSS \\ IGSO Inclined Geosynchronous Orbit \\ LEO Low Earth Orbit \\ LOS Line of Sight \\ MEO Medium Earth Orbit \\ MMS Magnetospheric Multiscale \\ MOD Maximum Outage Duration \\ MRTP Minimum Radiated Transmit Power \\ NavIC Navigation with Indian Constellation (NavIC) (India) \\ PVT Position, Velocity, and Time
}




$\begin{array}{ll}\text { QZSS } & \text { Quasi-Zenith Satellite System (QZSS) (Japan) } \\ \text { SSV } & \text { Space Service Volume } \\ \text { UN } & \text { United Nations } \\ \text { US } & \text { United States } \\ \text { WG-B } & \text { UN ICG Working Group B }\end{array}$

\section{REFERENCES}

1. O. Balbach, B. Eissfeller, G. Hein, T. Zink, W. Enderle, M. Schmidhuber, , and N. Lemke, "Tracking GPS above GPS Satellite Altitude: Results of the GPS Experiment on the HEO Mission Equator-S," Proceedings of the 9th International Technical Meeting of the Satellite Division of The Institute of Navigation (ION GPS 1996), Nashville, TN, September 1998.

2. T. Powell, P. D. Martzen, S. Sedlacek, C. Chao, R. Silva, A. Brown, and G. Belle, "GPS Signals in a Geosynchronous Transfer Orbit: Falcon Gold Data Processing," Proceedings of the Institute of Navigation National Technical Meeting, January 1999 , pp. 575-585.

3. G. Davis, M. Moreau, J. Carpenter, and F. Bauer, "GPS-Based Navigation and Orbit Determination for the AMSAT AO-40 Satellite," Proceedings of the Guidance, Navigation, and Control Conference, Reston, VA, August 2002.

4. M. Unwin, R. D. Van Steenwijk, P. Blunt, Y. Hashida, S. Kowaltschek, and L. Nowak, "Navigating Above the GPS Constellation - Preliminary Results from the SGR-GEO on GIOVE-A," Proceedings of the 26th International Technical Meeting of The Satellite Division of the Institute of Navigation (ION GNSS+ 2013), 2013, pp. 3305-3315.

5. J. Kronman, "Experience Using GPS For Orbit Determination of a Geosynchronous Satellite," Proceedings of the Institute of Navigation GPS, Salt Lake City, UT, September 2000.

6. Marareskul, D., "Space Service Volume and Russian GEO satellites PNT," United Nations International Committee on GNSS, Seventeenth Meeting of the Providers' Forum, Sochi, Russia, November $2016 . \quad$ URL: http://www.unoosa.org/pdf/icg/2016/icg11/pf17/pf1.pdf

7. Winternitz, Luke B., Bamford, William A., Price, Samuel R., "New High-Altitude GPS Navigation Results from the Magnetospheric Multiscale Spacecraft and Simulations at Lunar Distances," Proceedings of the 30th International Technical Meeting of The Satellite Division of the Institute of Navigation (ION GNSS+ 2017), Portland, Oregon, September 2017, pp. 1114-1126.

8. Winkler, A., Ramsey, G., Frey, C., Chapel, J., Chu, D., Freesland, D., Krimchansky, A., Concha, M., "'GPS Receiver On-Orbit Performance for the GOES-R Spacecraft," GNC 2017: 10th International ESA Conference on Guidance, Navigation \& Control Systems, Salzburg, Austria, May 2017.

9. Bauer, F., Moreau, M. C., Dahle-Melsaether, M., Petrofski, W., Stanton, B., Thomason, S., Harris, G., Sena, R., and Temple III, L. P., “The GPS Space Service Volume," Proceedings of the 19th International Technical Meeting of the Satellite Division of The Institute of Navigation (ION GNSS 2006), 2006, pp. 2503-2514.

10. United Nations, Office for Outer Space Affairs, "Global Navigation Satellite Systems: Education Curriculum," ST/SPACE/59 January

2013). http://www.unoosa.org/res/oosadoc/data/documents/2012/stspace/stspace59_0_html/st_space_59E.pdf

11. United Nations, Office for Outer Space Affairs, "International Committee on Global Navigation Satellite Systems: The Way $\quad$ Forward," ST/SPACE/67 (16 2016$)$ http://www.unoosa.org/res/oosadoc/data/documents/2016/stspace/stspace67_0_html/st_space_67E.pdf

12. Miller, J. J., Bauer, F. H., Oria, A.J., Pace, S., and Parker, J. J. K., "Achieving GNSS Compatibility and Interoperability to Support Space Users," Proceedings of the 29th International Technical Meeting of The Satellite Division of the Institute of Navigation (ION GNSS+ 2016), Portland, Oregon, September 2016, pp. 3622-3634.

13. United Nations, Office for Outer Space Affairs, "Report of Working Group: Enhancement of GNSS Performance, New Services and Capabilities," ICG/WGB/2015. http://www.unoosa.org/pdf/icg/2015/icg10/wg/wgb_report.pdf

14. United Nations, Office for Outer Space Affairs, "The Interoperable GNSS Space Service Volume," To be published 2018.

15. Enderle, W., Gini, F., Boomkamp, H., Parker, J. J. K., Ashman, B. W., Welch, B. W., Koch, M., and Sands, O. S., "Space User Visibility Benefits of the Multi-GNSS Space Service Volume: An Internationally-Coordinated, Global and Mission-Specific Analysis," Proceedings of the 31st International Technical Meeting of the Satellite Division of The Institute of Navigation (ION GNSS 2018), 2018.

16. Bauer, F. H.; Parker, J. J. K.; Welch, B., and Enderle, W., "Developing a Robust, Interoperable GNSS Space Service Volume (SSV) for the Global Space User Community," Proceedings of the 2017 International Technical Meeting of The Institute of Navigation, 2017. 
17. United Nations, Office for Outer Space Affairs, "Report of the Working Group B: Enhancement of Global Navigation Satellite Systems (GNSS) Services Performance," ICG/WGB/2014. http://www.unoosa.org/pdf/icg/2014/icg9/icg9_WGBreport.pdf

18. United Nations, Office for Outer Space Affairs, "Report of Working Group B: Enhancement of GNSS Performance, New Services and Capabilities, 9 and 10 November 2016, Sochi, Russian Federation," ICG/WGB/2016. http://www.unoosa.org/pdf/icg/2016/icg11/wgb/WGB2016report.pdf

19. United Nations, Office for Outer Space Affairs, "Report of Working Group B: Enhancement of GNSS Services Performance," ICG/WGB/2013. http://www.unoosa.org/pdf/icg/2013/icg-8/wgB/ICG_WGB_2013_report.pdf

20. Chapel, J., Stancliffe, D., Bevacqua, T., Winkler, S., Clapp, B., Rood, T., Freesland, D., Reth, A., Early, D., Walsh, T., Krimchansky, A., "In-Flight Guidance, Navigation, and Control Performance Results for the GOES-16 Spacecraft," GNC 2017: 10 ${ }^{\text {th }}$ International ESA Conference on Guidance, Navigation \& Control Systems, Salzburg, Austria, May 2017. 